\title{
Gestión del riesgo organizacional de fraude \\ y el rol de Auditoría Interna
}

\section{Mauricio De La Torre Lascano}

\author{
Facultad de Ciencias Administrativas \\ Universidad Central del Ecuador
}

El cambio vertiginoso en la tecnología ha dado lugar al crecimiento empresarial a nivel mundial y a toda escala, lo que implícitamente ha provocado que las organizaciones se enfrenten a nuevas contingencias. Factores exógenos, como la globalización o emisión de normativa internacional, y factores endógenos debido al incremento de operatividad han modificado totalmente la estructura organizacional clásica; como resultado, las organizaciones quedan expuestas a niveles sorprendentes de riesgo, incluido el de fraude. Los asiduos hechos que devienen de prácticas corruptas impactan no solo en las organizaciones afectadas por estos, sino en la sociedad y —en general— en el Estado donde estas ejecutan sus operaciones.

El objetivo de la presente investigación se basó en el análisis del rol de auditoría interna frente a la gestión del riesgo de fraude. A partir de este, por un lado, asume un enfoque cuantitativo del impacto económico en las organizaciones y, por otro, un enfoque cualitativo de los diferentes estándares internacionales emitidos, así como de diversos estudios doctrinales realizados sobre esta materia. Tanto los estándares internacionales como la legislación emitida por las naciones buscan incrementar la credibilidad de la información reportada a los entes reguladores, robustecer mecanismos preventivos y fomentar la responsabilidad de las organizaciones por la gestión del riesgo de fraude. No obstante, quedan pendientes aún varios temas por legislar, como, por ejemplo, la imperativa adopción de dicha normativa internacional y la implementación obligatoria de una unidad independiente de auditoría interna en todas las compañías, que contribuya a generar valor y robustecer la transparencia en las mismas.

Palabras clave: corrupción, estándares internacionales, fraude, globalización, transparencia

\section{Management of organizational fraud risk and Internal Audit role}

Vertiginous change in technology has led to global and full-scale business growth, which implicitly has caused organizations to face new contingencies, exogenous factors such as globalization or issue of international regulations and endogenous factors due to the increase in operations, have completely modified the classical organizational structure, resulting exposed to surprising levels of risk, including fraud. The frequent facts that come from corrupt practices impact not only the organizations where they are committed, but the society and in general the State where they execute their operations. The objective of the current research was based on the analysis of the internal audit role against the fraud risk management with a quantitative approach of the economic impact in the organizations and qualitative of the different international standards issued, as well as of diverse doctrinal studies made on this matter. Both the international standards and the legislation issued by the nations seek to increase the credibility of the information reported to the regulatory bodies, strengthen preventive mechanisms and encourage the organizations' responsibility for fraud risk management. However, there are still pending issues for legislation, such as the imperative adoption 
of such international regulations and the mandatory implementation of an independent Internal Audit unit in all companies that contributes to generate value and strengthen transparency in them.

Keywords: Corruption, international standards, fraud, globalization, transparency

\section{Gestão do risco de fraude organizacional e papel de Auditoria Interna}

A rápida mudança na tecnologia levou ao crescimento dos negócios em todo o mundo e cada escala, o que implicitamente significa que as organizaçôes estão enfrentando novas contingências, fatores exógenos tais como a globalização ou emissão de normas internacionais e fatores endógenos devido ao aumento Operabilidade, modificaram completamente a estrutura organizacional clássica, resultando exposta a níveis surpreendentes de risco, incluindo fraude. Os fatos freqüentes que vêm de práticas corruptas impactam não apenas as organizaçôes onde estão comprometidos, mas a sociedade e em geral o Estado onde executam suas operaçóes. O objetivo desta pesquisa foi baseada na análise do papel da auditoria interna contra o risco de fraude de gestão com um impacto econômico quantitativa sobre as organizaçôes e da qualidade das diversas normas internacionais abordagem emitido e vários estudos doutrinários feita sobre este assunto. Tanto as normas internacionais quanto a legislação emitida pelas naçôes buscam aumentar a credibilidade das informaçôes reportadas aos órgáos reguladores, fortalecer os mecanismos preventivos e incentivar a responsabilidade das organizaçôes pelo gerenciamento do risco de fraude. No entanto, várias questôes ainda precisam ser legisladas, como a adoção imperativa de tais regulamentaçôes internacionais e a implementação obrigatória de uma unidade de Auditoria Interna independente em todas as empresas que contribuem para gerar valor e fortalecer a transparência nelas.

Palavras-chave: corrupção, padróes internacionais, fraude, globalização, transparência

\section{Introducción}

Las empresas han sido creadas con la finalidad de obtener el máximo de rentabilidad con el uso eficiente de sus limitados recursos, es decir, buscando la manera de optimizar sus actividades operacionales. Sin embargo, lamentablemente, en muchas ocasiones, han utilizado artilugios contables para lograr esa consecución de beneficios. Maldonado (2016) determina una diferencia entre una situación en que una persona invierte su capital y esfuerzo personales en un negocio para obtener su justa ganancia (empresarios), y otra en que la empresa contrata ejecutivos que se encarguen de administrarla para beneficio de sus accionistas. En estos casos, el administrador pone en juego todas sus habilidades y capacidades gerenciales para que la empresa gane dinero, y ese ejecutivo sea evaluado y remunerado en función de esos resultados económicos, a veces, sin importar el impacto o las consecuencias de tales actos. Esa búsqueda de eficiencia por parte de las organizaciones ha estado presente desde siempre. Así, por ejemplo, Soto (2016) determina que en la Revolución Industrial se llevó a cabo una transformación económica, social y tecnológica en el Reino Unido a mitad del siglo XVIII; décadas después, se extendió a gran parte de Europa occidental y Norteamérica, y concluyó entre 1820 y 1840. Durante ese proceso, el mundo pasó de una economía rural basada fundamentalmente en la agricultura y el comercio a una economía de carácter urbano, industrializada y automatizada.

Utilizando una óptica positiva, Buscaglia, González, Fumarulo y Prieto afirman que «[...] la globalización está presente en la realidad cotidiana del actuar humano, en todos los aspectos, representando grandes oportunidades para hacer negocios lícitos, haciendo crecer las economías y las oportunidades de desarrollo 
para los ciudadanos y las naciones» (2002, p. 2). A partir de ello, realzan los beneficios de este proceso de integración mundial que, entre otras cosas, permite obtener información en línea uniendo a los habitantes de cualquier región y a las sociedades jurídicas, con lo cual desaparece cada vez más el limitante que anteriormente la distancia y las fronteras físicas establecían.

En un ambiente empresarial global cada vez más estandarizado, el cumplimiento normativo resulta imprescindible; de hecho, en la actualidad, iniciar actividades comerciales sin una estrategia claramente definida es una odisea que muy pocos inversionistas, inclusive inexpertos, realizarían. «Por los cambios tecnológicos, las aplicaciones económicas y por la llamada globalización de la economía, las organizaciones han venido evolucionando rápidamente y por ello los riesgos y, por ende, los controles han tenido que modificarse» (Estupiñán, 2006, p. 11). En esa vertiginosa transición carente de controles en la que se han visto inmersas casi sin darse cuenta las compañías, se han generado ambientes propicios para que la delincuencia criminal organizada engendre prácticas corruptas. Entre ellas, resalta el fraude corporativo, con especial referencia a los escándalos mundiales protagonizados por multinacionales como Odebrecht, Worldcom, Enron, Parmalat o Volkswagen. Al respecto, Ryder indica: «el fraude ha emergido recientemente de la sombra de lavado de dinero y el financiamiento del terrorismo tras varios casos de alto perfil de las actividades fraudulentas de las empresas multinacionales» (2011, p. 1).

Se debe tomar en consideración que las corporaciones transnacionales poseen mucho poder económico y político donde desarrollan sus actividades, ya sea a nivel nacional, regional y mundial. En ciertos casos, incluso, cuentan con la capacidad para manipular información contable y evadir fácilmente controles establecidos. Los auditores no son culpables de adulterar las cuentas; las cuentas las adulteran los directivos y los administradores de las empresas, y, en este sentido, una de las cuestiones más polémicas y que subyace en el trasfondo de los escándalos financieros es el hecho de que se percibe en ellos una cierta concentración de poder. Específicamente, «En reiterados casos es el poder omnímodo de los mayores accionistas el que fuerza los maquillajes contables» (García y Vico, 2003, p. 29).

Black (2005) determina que individualmente los fraudes de control causan mayores pérdidas que todas las otras formas de delitos contra la propiedad combinadas; por lo tanto, podrían ser calificados como «superdepredadores financieros». Como muestran los recientes casos de fraudes de control, se observa que son crímenes dirigidos por el jefe de Estado o el director general de una compañía que usa la nación o la compañía como un vehículo del fraude. Las olas de fraude de control pueden causar colapsos económicos; dañar y desacreditar instituciones clave vitales para una buena gobernanza política; y, como consecuencia, erosionar la confianza. El elemento determinante del fraude es el engaño: el criminal crea y, luego, traiciona la confianza. El fraude, por ende, es el ácido más fuerte para corroer la confianza. En el caso concreto del fraude de control, la consecuencia es que las instituciones y la confianza se vuelven frágiles — se desmoronan-, lo cual produce un estancamiento económico.

Presentado el entorno actual en que las empresas desarrollan sus actividades, se infiere la necesidad de establecer técnicas de prevención y detección de fraude apropiadas, a cargo de una unidad independiente de aseguramiento que ayude a mermar el riesgo empresarial que incluye dantescas pérdidas financieras por daños irreparables, reputacionales y sociales.

Contabilidad y Negocios (13) 25, 2018 / ISSN 1992-1896 


\section{Metodología}

El presente trabajo de investigación se desarrolló, por un lado, sobre la base de un análisis cuantitativo del impacto económico del fraude en las organizaciones, y, por otro, uno cualitativo de los diferentes estándares internacionales emitidos por organismos mundiales reconocidos que buscan incrementar la credibilidad de la información reportada a los entes reguladores, robustecer mecanismos preventivos y fomentar la responsabilidad de las organizaciones por la gestión del riesgo de fraude. Se utilizaron varias fuentes documentales, especialmente, estudios realizados por firmas internacionales de auditoría especializadas en el tema, así como estudios académicos e investigaciones para determinar dicho impacto. Aunque existe vasto cúmulo de información sobre fraude, el análisis se ha enfocado sobre aquella que establece una relación entre la gestión del riesgo organizacional de fraude y la importancia de la auditoría interna. Esta selección responde a que ello constituye la base del objetivo central de la investigación, que determina la necesidad de adopción de estándares internacionales y la exigencia de su aplicación global debido a diferentes sucesos acaecidos que tienen como constante la actividad criminal. Es necesario acotar que, al tratarse de un estudio cualitativo documental, la técnica del análisis basada en la recopilación de doctrina y normativa internacional es la más idónea.

\section{Resultados y discusión}

Puesto que el objetivo general que buscó la investigación radica en el análisis de la importancia del rol de Auditoría Interna frente a la gestión del riesgo de fraude, mediante la necesidad de adopción de estándares internacionales y la exigencia de su aplicación global, se procedió al análisis detallado en esta sección.

\subsection{Fraude}

El fraude es un fenómeno complejo sobre el cual existe un vasto análisis tanto desde lo académico como desde la vivencia operacional propiamente dicha de las organizaciones en las que este fenómeno se presenta cada vez con más frecuencia; a pesar de ello, su estudio aún no está concluido o resuelto, puesto que su esencia es inherente a la naturaleza humana. En relación con ello, García y Humphrey señalan: «La responsabilidad del auditor ante el fraude empresarial es uno de los temas más polémicos y controvertidos existentes en la historia de la auditoría. Durante el último siglo, la función asignada al auditor frente al fraude no ha sido constante» (1995, p. 697). Esta visión o enfoque ha sufrido grandes y múltiples modificaciones, acorde con las diferentes etapas históricas. Se ha adoptado posiciones totalmente contrarias entre sí, especialmente, desde sus inicios hasta nuestros días. Frente a ese contexto, «Es importante acotar que el objetivo del control interno o de cualquier auditoría interna o externa no es detectar fraudes como comúnmente se le solía asociar» (Berbia, 2008, p. 78).

The Institute of Internal Auditors define el fraude de la siguiente manera: «[...] cualquier acto ilegal caracterizado por engaño, ocultación o violación de confianza. Estos actos no requieren la aplicación de amenaza de violencia o de fuerza física. Los fraudes son perpetrados por individuos y por organizaciones para obtener dinero, bienes o servicios, para evitar pagos o pérdidas de servicios, o para asegurarse ventajas personales o de negocio» (2017b, p. 61). Debido a sus diferentes modalidades y metodologías utilizadas para perpetrarlo, el fraude deviene en un concepto legal amplio. En el contexto de la auditoría de estados financieros o auditoría financiera, este se define como un error intencional en los estados financieros. "Las dos principales categorías de fraude son: informes financieros 
fraudulentos y malversación de activos» (Arens, Elder y Beasley, 2007, p. 314).

En cuanto a la información financiera fraudulenta, esta es entendida, según el Informe Treadway, como la «[...] conducta intencional o imprudente, ya sea por acción u omisión, que desemboca en la distorsión de estados financieros» (AICPA, 1987, p. 2). La información financiera fraudulenta puede involucrar muchos factores endógenos o exógenos, y tomar muchas formas. Supone una distorsión grave y deliberada de los registros corporativos, como marcas de inventario o transacciones falsificadas, como ventas o pedidos ficticios. Puede implicar, también, una aplicación errónea de principios o normas internacionales de contabilidad. En este turbio proceso, pueden estar involucrados funcionarios de la empresa de cualquier nivel, desde la Alta Dirección hasta niveles operativos. A partir de ello, justamente, nace la importancia de que las organizaciones cuenten con un sistema de control interno integral y robusto; en la medida que, en muchas ocasiones, los controles adoptados e implementados pueden resultar ineficaces ante el fraude, «los altos directivos pueden sobrepasar los controles. Un alto directivo o gerente que busque realizar un gran fraude puede sobrepasar los controles internos establecidos» (Cano, 2006, p. 374).

Para poder entender el complejo esquema de la corrupción en el ámbito organizacional, es necesario diferenciar un acto corrupto de otras actividades nocivas similares. La diferenciación con el fraude radicaría en la intencionalidad para obtener un beneficio económico. Dentro de ese marco, la corrupción organizacional de individuos podría ir desde sobornos, distorsión de información financiera, sobreestimación de gastos u horas trabajadas, fraude e -inclusorobo. Cuando la corrupción organizacional se realiza en beneficio de la organización, puede manifestarse como fraude corporativo, crimen de negocios y desviación del negocio hacia actividades criminales (Arellano, 2017, p. 815).

Tomando en consideración el impacto negativo que genera el fraude en las organizaciones, la participación directa de auditoría interna con un enfoque preventivo no solo resulta muy necesario, sino esencial para mermar las posibilidades que este ocurra o que se generen las condiciones propicias para ello. Es decir, la aplicación de un sistema preventivo siempre será útil para tal fin. Dentro de esta perspectiva, también, es importante la disuasión, puesto que se puede convencer a los funcionarios que no cometan fraude. Sobre este punto, Rozas precisa que «Tanto la prevención como la disuasión son menos costosas que el tiempo y el gasto requerido para la investigación y detección del fraude cuando ya fue cometido» (2009, p. 68).

Para que la prevención sea efectiva, debe entenderse que el sistema de control interno es responsabilidad de todos los miembros de la organización sin tomar en consideración el nivel jerárquico al que correspondan. COSO determina que «[...] un sistema de control interno efectivo requiere algo más que un riguroso cumplimiento de las políticas y procedimientos: requiere del juicio y del criterio profesional. La dirección y el consejo de administración deben utilizar su criterio profesional para determinar el nivel de control que es necesario aplicar» (2013, p. 1).

En cuanto a los individuos que lo perpetran, PricewaterhouseCoopers determina que, detrás de todas estas tipologías de fraude cada vez más habituales en las organizaciones, se esconden defraudadores con las siguientes características (2016, p. 33):

- Forman parte de la estructura interna de las compañías.

Contabilidad y Negocios (13) 25, 2018 / ISSN 1992-1896 
- Cometen un fraude como consecuencia de la existencia de un entorno favorable para cometer actos irregulares.

- Son hombres entre los 41 y los 50 años, con estudios superiores y que llevan más de 10 años trabajando en las organizaciones.

- Ocupan un cargo intermedio o son miembros de la Alta Dirección.

En referencia al costo organizacional del fraude calculado en diversas organizaciones a nivel mundial, el estudio de Gee y Button (2015) determina que el porcentaje global de pérdidas en utilidades promedio por fraude es del 5,6\%. Entre sus conclusiones, los autores resaltan tres puntos que están claros:

- Las pérdidas por fraude y error se pueden medir, y de manera efectiva.

- Sobre la base de las evidencias, es probable que las pérdidas en cualquier organización y cualquier área de gasto correspondan, por lo menos, con el 3\%; probablemente, cerca del $6 \%$; y, posiblemente, más del $10 \%$.

- Con el beneficio de información precisa sobre su naturaleza y extensión, pueden reducirse significativamente (Gee y Button, 2015).

La Asociación de Examinadores de Fraude Certificados (ACFE, 2016) ha realizado una clasificación del fraude y abuso ocupacional que data de 1996, tomando en consideración varios patrones en la forma en que se cometían los ilícitos. Una vez organizados los casos de acuerdo con esos patrones, determinaron que casi todos los esquemas de fraude ocupacional caían en categorías específicas, las cuales atacaban diferentes funciones y operaciones dentro de las organizaciones. Sobre la base de esas categorías, generaron un sistema completo para clasificar los esquemas de fraude ocupacional a fin de ayudar a las organizaciones a entender sus riesgos de fraude y, consecuentemente, a generar controles antifraude específicos. Son tres los patrones determinados: corrupción, apropiación ilegal de activos y fraude en estados financieros.

El ACFE define al fraude ocupacional como «[...] el uso deliberado de la ocupación para el enriquecimiento personal, mediante el mal uso o desvío de los recursos o activos de la organización contratante» (2016, p. 6). En el sector privado, el perpetrador sustrae a los acreedores de la compañía (entre los cuales se incluyen los trabajadores) y a los accionistas. Un ejemplo clásico es aquel en que el director general lo utiliza para exagerar los beneficios y el patrimonio neto de la organización para darles a los activos de la empresa uso personal a través de mecanismos corporativos aparentemente normales y legítimos (por ejemplo, sueldos, bonos y opciones sobre acciones) (Black, 2005).

El Reporte a las Naciones sobre el abuso y el fraude ocupacional elaborado por la ACFE (2016) provee un análisis de 2410 casos de fraude ocupacional que ocurrieron en 114 países alrededor del mundo, y que fueron investigados entre enero 2014 y octubre 2015. El informe incluye un análisis sobre el costo del fraude y señala el impacto económico del mismo a nivel global. De acuerdo con su análisis, los 2410 casos causaron una pérdida total de más de US\$ 6300 millones (ACFE, 2016). La pérdida media de un caso en particular de fraude ocupacional fue de US\$ 150.000. Considerando que el fraude es costoso y la detección del fraude es compleja, la identificación de métodos para reducir el fraude debería ser de suma importancia para las partes interesadas (stakeholders), incluidos accionistas, instituciones financieras y clientes (Fathil y Schmidtke, 2010). Ello implica la creación o fortalecimiento de una unidad independiente que permita 
evaluar o implementar de manera oportuna (ex-ante) programas de prevención de fraude.

\subsection{Gestión del riesgo organizacional de fraude}

Nieto (2014) determina que el análisis de riesgos es una herramienta fundamental para confeccionar un programa de cumplimiento normativo eficaz. Sirve precisamente para indicar a la empresa cuáles son las obligaciones legales más importantes a las que está sujeta y dónde se sitúan sus puntos débiles. Igualmente, la complejidad en la elaboración de un programa de cumplimiento normativo depende del tamaño de la empresa y su estructura orgánica funcional. En las economías desarrolladas, es habitual que las compañías se encuentren cotizadas en la bolsa de valores. La robusta regulación en esos mercados de valores y del derecho de esas sociedades obliga a contar con órganos internos, a nivel de unidad administrativa o como nivel asesor mediante comités de auditoría o de riesgos, que deben conectarse con el sistema de cumplimiento normativo. Resulta necesario destacar que, a pesar de existir estándares internacionales que han aterrizado en la formulación de códigos de ética, responsabilidad social empresarial, gobierno corporativo y otros, si existen conflictos de interés en los que primen intereses personales en las relaciones comerciales, el objetivo de controlar el fraude corporativo se torna en extremo complejo, puesto que se pondrían de «[...] manifiesto la fatal atracción y superioridad del poder y la ambición económica frente a los tradicionales más aparentes que reales - criterios de honorabilidad en el campo profesional y laboral y frente a la ética de los negocios en general» (Carbajo, 2012, p. 289).

Bajo estos preceptos, se entiende la adopción obligatoria del control, la supervisión y la generación de valor en todas las actividades administrativas o inherentes al proceso productivo de las compañías, especialmente, de aquellas catalogadas como pymes, ya sea mediante la implantación de un departamento o área de Auditoría Interna, o bien con el fortalecimiento de dicha área, que —además de ejercer control en todas y cada una de las actividades y procesos productivos que posee- genere valor a los mismos, y aporte de manera proactiva y eficiente a la Administración de la compañía (De La Torre, 2009, p. 23). El valor en las organizaciones se maximiza cuando «[...] la dirección establece una estrategia y objetivos para encontrar un equilibrio óptimo entre los objetivos de crecimiento y rentabilidad y los riesgos asociados, además de desplegar recursos efectiva y eficientemente a fin de lograr los objetivos de la entidad» (COSO, 2005, p. 3).

En ese contexto, entra a colación la auditoría interna. The Institute of Internal Auditors define la auditoría interna como una "[...] actividad independiente y objetiva de aseguramiento y consulta, concebida para agregar valor y mejorar las operaciones de una organización. Ayuda a una organización a cumplir sus objetivos aportando un enfoque sistemático y disciplinado para evaluar y mejorar la eficacia de los procesos de gestión de riesgos, control y gobierno" (2017a). Ello implica que esta unidad debe proveer aseguramiento a la organización respecto a si los controles que tienen implementados son apropiados en función del apetito de riesgo que posee la organización. En otras palabras, la auditoría interna debería proporcionar un aseguramiento objetivo al consejo de Administración y a la Gerencia en cuanto a si los controles antifraude son suficientes para los riesgos de fraude identificados, $y$ asegurar que estos se encuentren funcionando con eficacia.

Los auditores internos pueden revisar la amplitud e idoneidad de los riesgos, especialmente, aquellos con respecto a la evasión de controles por parte de la Gerencia (IIA, 2008). En relación con ello, Halbouni (2015) 
señala que las unidades de Auditoría Interna son fundamentales para prevenir el fraude: constituyen la fuerza central para descubrir o limitar la apropiación indebida de activos y los esquemas de corrupción. A pesar del hecho de que los auditores internos no siempre supervisan los programas de prevención de fraude, es lógico que participen en la gestión del riesgo organizacional de identificación de dichos riesgos, y en el diseño de programas de monitoreo y supervisión para reducirlos.

La importancia que una organización incorpore a sus actividades una unidad de auditoría interna constituye un indicio del compromiso que tiene la organización para realizar un control interno efectivo y un manejo del riesgo de fraude. Los auditores internos pueden realizar auditorías proactivas con el fin de buscar casos de malversación de activos y de presentación de información falsa. Esto puede incluir el uso de técnicas de auditoría asistidas por computador (TAAC), incluida la minería de datos (data mining), con el fin de detectar tipos particulares de fraude. Pueden, también, aplicar procedimientos analíticos y de otra índole, que les permita encontrar rubros inusuales, y llevar a cabo análisis detallados de cuentas y transacciones de alto riesgo para identificar un posible fraude (IIA, 2009).

Con respecto a ello, Kagermann, Kinney, Kuting y Weber (2007) determinan que los fundamentos de auditoría interna deben proporcionar una base sólida para el concepto general resultante. Las reglas contenidas en el código de conducta proporcionan el marco para todas las actividades significativas realizadas por auditoría interna para garantizar el cumplimiento en particular y el gobierno corporativo en general. Deben incluirse las pautas para cumplir con numerosas leyes, normativas, actos y regulaciones. Todas estas pautas deben almacenarse en una base de datos en formato de texto, así como tablas de verificación para documentar el grado de cumplimiento. En este sentido, además, puede haber enlaces basados en el sistema entre las tablas de verificación y los tipos de auditoría individuales, de modo que se pueda proporcionar simultáneamente evidencia de que las reglas definidas allí se han implementado realmente en el sistema.

«Los controles antifraude son aquellos diseñados por la administración para prevenir, detectar y/o corregir los fraudes» (IFAC, 2007, p. 12). Si bien de acuerdo con la capacidad que tiene la Administración para eludir los controles estos pueden no prevenir que ocurra el fraude, al menos, pueden actuar como elementos disuasivos y hacen más difícil cometerlo. A continuación, se mencionan algunos ejemplos típicos:

- Políticas y procedimientos que proveen aseguramiento (accountability) adicional, tales como las aprobaciones firmadas de las entradas en registros contables

- Controles de acceso mejorados para los datos y las transacciones sensibles

- Alarmas silenciosas

- Reportes de discrepancia y excepción

- Rastros de auditoría

- Planes de contingencia por fraude

- Procedimientos de recursos humanos, tales como identificación/monitoreo de individuos con potencial de fraude anterior-promedio (por ejemplo, un estilo de vida excesivamente lujoso)

- Mecanismos para reportar anónimamente los fraudes potenciales

Los riesgos de fraude y la capacidad de la Administración para anular el control interno están presentes en 
todas las organizaciones. El principal responsable del diseño, implementación y mantenimiento del control interno es el consejo de Administración (directorio), seguido de la Gerencia, por lo que la entidad siempre está expuesta al peligro de que la Administración anule los controles, ya sea en una entidad pública, privada, sin fines de lucro o gubernamental. Cuando la oportunidad de anular el control interno se combina con poderosos incentivos para cumplir con los objetivos contables, la Alta Dirección puede participar en informes financieros fraudulentos (AICPA, 2016). Por ello, "[...] el comportamiento de la administración puede tener un gran impacto en las percepciones externas de la identidad de una organización» (Sitorus y Scott, 2009, p. 288). La Administración puede anular los controles para declarar de manera errónea intencionalmente la naturaleza, y la oportunidad de los ingresos $\mathrm{u}$ otras transacciones, mediante tres estrategias. En primer lugar, puede registrar acontecimientos o transacciones de negocios ficticios, o cambiar el momento del reconocimiento de transacciones legítimas, particularmente, aquellas registradas cerca del final del período contable. En segundo lugar, puede establecer o revertir reservas para manipular resultados; incluso, intencionadamente, las suposiciones y los juicios sesgados utilizados para estimar saldos de cuentas. Finalmente, puede alterar registros y términos relacionados con transacciones significativas o inusuales.

Todas las organizaciones están sujetas a riesgos de fraude. Es imposible eliminar totalmente el fraude en todas las organizaciones. Sin embargo, se han establecido principios con la finalidad de que el fraude sea prevenido o detectado de manera oportuna y se cree un efecto robusto de disuasión del fraude. Por ejemplo, COSO (2016) ha emitido cinco principios en la gestión del riesgo organizacional de fraude:
- La organización establece y comunica un programa de gestión del riesgo de fraude que demuestra las expectativas de la Junta Directiva y la Alta Dirección, y su compromiso con la alta integridad y valores éticos en relación con la gestión de control de riesgo de fraude.

- La organización realiza evaluaciones exhaustivas en la gestión del riesgo de fraude para identificar esquemas y riesgos específicos de fraude, evaluar su probabilidad e impacto, evaluar las actividades de control de fraude existentes y ejecutar acciones para mitigar los riesgos residuales de fraude.

- La organización selecciona, desarrolla e implementa actividades preventivas y de detección de fraude para mitigar el riesgo de que ocurran eventos de fraude o que no sean detectados de una manera oportuna.

- La organización establece un proceso de comunicación para obtener información sobre potenciales fraudes, e implementa un enfoque coordinado para la investigación y la acción correctiva para abordar el fraude apropiadamente y de forma oportuna.

- La organización selecciona, desarrolla y realiza evaluaciones continuas para determinar si cada uno de los cinco principios de gestión del riesgo de fraude está presente y funcionando. Además, comunica las deficiencias de manera oportuna del Programa de Gestión del Riesgo de Fraude a las partes que son responsables de tomar medidas correctivas, incluidas la Gerencia Senior y la Junta Directiva.

Si bien en el desarrollo de este estudio se ha analizado en gran medida principios aplicables al sector privado, que es en el cual las organizaciones desarrollan sus actividades, el fenómeno del fraude también está presente en el sector público. Así, el U.S. Government 
Accountability Office (GAO) acota que «[...] el objetivo de la gestión del riesgo de fraude es asegurar la integridad del programa mediante la mitigación continua y estratégica de la probabilidad y el impacto del fraude» (2015, p. 5). En función de dicho objetivo, determina que las actividades de control crítico para gestionar los riesgos de fraude se dividen en tres categorías generales: prevención, detección y respuesta. Estas categorías son interdependientes y se refuerzan mutuamente. A partir de ello, GAO ha desarrollado un marco de referencia para la gestión de riesgo de fraude en programas federales estableciendo cuatro componentes:

- Compromiso: Comprometerse a combatir el fraude mediante la creación de una cultura organizativa y una estructura conducentes a la gestión del riesgo de fraude

- Evaluación: Planificar las evaluaciones regulares del riesgo de fraude y evaluar los riesgos para determinar un perfil de riesgo de fraude

- Diseño e implementación: Diseñar e implementar una estrategia con actividades de control específicas para mitigar los riesgos de fraude evaluados y colaborar para ayudar a asegurar una implementación efectiva

- Evaluación y adaptación: Evaluar los resultados utilizando un enfoque basado en riesgos y adaptar las actividades para mejorar la gestión del riesgo de fraude

\section{Conclusiones}

El fraude causa impactos negativos en todas las organizaciones no solo de orden económico, sino también reputacional y social, tanto en el sector público como en el sector privado. La teoría del fraude de control explica por qué los fraudes causan pérdidas tan desproporcionadas. En el sector privado, cualquier individuo que lo comete es el perpetrador. Cuando se utiliza a la nación como un vehículo de fraude, se le denomina cleptocracia. Se pueden clasificar los fraudes de control del sector privado por la naturaleza de la víctima principal intencionada del fraude. En este sector, el perpetrador sustrae los bienes a los acreedores de la compañía (que incluyen a los trabajadores) y a los accionistas (Black, 2005).

La función de control es una de las dimensiones más relevantes de la estructura de gobierno corporativo de las organizaciones. Integra en su ámbito el control interno, cuya amplitud en su misión abarca objetivos, planes estratégicos, políticas, procedimientos, procesos, sistemas, actividades, funciones, proyectos, iniciativas y emprendimientos de todos los tipos y en todos los niveles de una organización. Entre ellos, se incluyen aquellos intangibles que influencian la cultura organizacional (Montenegro, Oliveira y Lopes, 2018), para lo cual resulta necesaria esa interacción (con énfasis en la supervisión) que debe ejercer la unidad de Auditoría Interna con todos los miembros de la organización.

La prevención que debe ser diseñada de manera global por Auditoría Interna desempeña una función vital dentro de la gestión de riesgo de fraude, pues, si bien los principios, políticas, procedimientos adoptados no aseguran de manera fehaciente que este no ocurra, promueve en toda la organización una cultura positiva de los valores éticos y difunde los graves impactos de toda índole que podrían suceder si se presentasen. Este proceso de integridad es muy importante en todos los niveles jerárquicos y de dirección para aumentar la conciencia de las consecuencias de la corrupción, mejorar la comprensión de algunos de los principios éticos aplicables, pero también cultivar un 
ethos general que involucre auténticos compromisos morales, los derechos humanos y las preocupaciones ambientales, en los negocios y en la política (Roy y Singer, 2006). La visión empresarial típica, según la cual no se implementa acciones preventivas en torno al fraude en tanto no ocurran los nefastos eventos, es muy común, sin considerar que «[...] en el contexto económico actual, no tener en cuenta los beneficios financieros de hacer reducciones relativamente fáciles de las pérdidas de fraude y error parece temerario» (Gee y Button, 2015, p. 11).

Si bien la auditoría a los estados financieros emite una opinión acerca de la razonabilidad de los estados financieros, esta normativa se está ampliando, y abarcando tópicos y temáticas inherentes a la correcta administración de riesgos: «en los últimos años algunos países están exigiendo dentro de la auditoría financiera, la opinión sobre el control interno financiero, lo cual constituye un acercamiento a la auditoría integral» (Blanco, 2012, p. xvi). Existen estándares internacionales que han sido emitidos por organismos mundialmente reconocidos como principios y marcos de referencia que coadyuvan a que la gestión del riesgo de fraude sea adoptada con una visión proactiva, lo cual minimiza el impacto que pudiera generarse. «El resultado de la correcta aplicación por parte de las organizaciones de estándares internacionales, mediante una unidad independiente de aseguramiento y consulta, trae como resultado que exista mayor transparencia en el accionar operativo y confiabilidad en la información remitida» (De La Torre, 2017, p. 123). De hecho, se ha emitido variada normativa de reciente data por organismos internacionales para su aplicación, tanto en el sector público como en el sector privado, que debería ser de obligatoria implementación (como, por ejemplo, la literatura sobre gobierno corporativo emitida por la OECD, códigos de conducta, ISO 37001, AS/ NZS, entre otros). Su finalidad no solamente radica en proveer de un nivel de aseguramiento adecuado en las organizaciones, precepto que va en línea con el espíritu de auditoría interna, sino que también aporta en la generación de valor. De esta manera, afronta técnicamente los riesgos de fraude y contribuye con el cumplimiento de los objetivos institucionales que radican en la consecución de rentabilidad.

\section{Referencias bibliográficas}

American Institute of Certified Public Accountants AICPA (1987). Report of the National Commission on Fraudulent Financial Reporting, Treadway Commission. Nueva York: AICPA. Consulta: 30 de abril de 2018. https:// www.coso.org/Documents/NCFFR.pdf

American Institute of Certified Public Accountants AICPA (2016). Management override of internal control: The Achilles' Heel of fraud prevention. Nueva York: AICPA. Consulta: 30 de abril de 2018. http://www.aicpa.org/ ForThePublic/AuditCommitteeEffectiveness/DownloadableDocuments/achilles_heel.pdf

Arellano, D. (2017). Corrupción como proceso organizacional: Comprendiendo la lógica de la desnormalización de la corrupción. Contaduría y Administración, 62, 810-826. Consulta: 15 de abril de 2018. https://doi. org/10.1016/j.cya.2016.01.005

Arens, A., Elder, R. y Beasley, M. (2007). Auditoría. Un enfoque integral. México: Pearson.

Association of Certified Fraud Examiners (ACFE) (2016). Reporte a las naciones sobre el abuso y el fraude ocupacional. Austin: ACFE. Consulta: 17 de abril de 2018. http://acfe-mexico.com.mx/archivos/Reporte_Naciones_2016_esp.pdf

Berbia, P. (2008). Evaluación eficaz del sistema de control interno. Altamonte Springs: Institute of Internal Auditors Research Foundation.

Contabilidad y Negocios (13) 25, 2018 / ISSN 1992-1896 
Black, W. K. (2005). When fragile becomes friable: Endemic control fraud as a cause of economic stagnation and collapse. En White Collar Crimes: A Debate (pp. 162-178). Hyderabad, India: The ICFAI University Press. Consulta: 5 de mayo de 2018. http://ssrn. com/abstract $=1536528$

Blanco, Y. (2012). Auditoría integral: normas y procedimientos. Bogotá: Ecoe Ediciones.

Buscaglia, E., González, S., Fumarulo, S. y Prieto, C. (2002). Delincuencia organizada y terrorismo: $\mathrm{Su}$ combate a través de la Convención de Palermo. Revista Universitaria, 1(1), 1-9. Consulta: 5 de mayo de 2018. http://ssrn.com/abstract $=946943$

Cano, M. (2006). Auditoría forense aplicada en investigaciones de corrupción administrativa. Control interno y fraudes (pp. 343-357). Bogotá: Ecoe Ediciones.

Carbajo, F. (2012). Corrupción en el sector privado (I): La corrupción privada y el derecho privado patrimonial. Iustitia, 10, 281-342. Consulta: 5 de mayo de 2018. https://doi.org/10.15332/iust.v0i10.885

Committee of Sponsoring Organization of the Treadway Commission (COSO) (2005). Administración de riesgos corporativos. Marco integrado. Jersey City: COSO.

Committee of Sponsoring Organization of the Treadway Commission (COSO) (2013). Control interno. Marco integrado. Resumen ejecutivo. Jersey City: COSO.

Committee of Sponsoring Organization of the Treadway Commission (COSO) (2016). Frand Risk Management Guide. Executive summary. Jersey City: COSO. Consulta: 17 de abril de 2018. https://www.coso.org/ Documents/COSO-Fraud-Risk-Management-GuideExecutive-Summary.pdf

De La Torre, M. (2009). Introducción a la auditoría interna. El Emprendedor, 3(5), 22-25.

De La Torre, M. (2017). Responsabilidad social corporativa y auditoría interna, una exigencia global imperativa. Revista Metanoia, 3(3), 113-126.
Estupiñán, R. (2006). Control interno y fraudes. Bogotá: Ecoe Ediciones.

Fathil, F. y Schmidtke, J. (2010). The relation between individual differences and accountants' fraud detection ability. International Journal of Auditing, 14(2), 163-173. Consulta: 17 de abril de 2018. https://doi. org/10.1111/j.1099-1123.2009.00412.x

García, M. A. y Humphrey, C. (1995). La auditoría y el fraude: algunas consideraciones internacionales. Revista Española de Financiación y Contabilidad, 24(84), 697-725. Consulta: 18 de abril de 2018. http://aeca. es/old/refc_1972-2013/1995/84-4.pdf

García, M. A. y Vico, A. (2003). Los escándalos financieros y la Auditoría: pérdida y recuperación de la confianza en una profesión en crisis. Revista Valenciana de Economia y Hacienda, 7. Consulta: 17 deabrilde2018. https:/www. researchgate.net/profile/Maria_Garcia_Benau/publication/28061516_Los_escandalos_financieros_y_ la_auditoria_perdida_y_recuperacion_de_ la_confianza_en_una_profesion_en_crisis/ links/00b7d5229bec968478000000.pdf

Gee, J. y Button, M. (2015). The financial cost of fraud 2015. What the latest data from around the world shows. Londres: PKF Littlejohn LLP. Consulta: 18 de abril de 2018. http://www.pkf.com/media/31640/PKF-Thefinancial-cost-of-fraud-2015.pdf

Halbouni, S. S. (2015). The role of auditors in preventing, detecting, and reporting fraud: The case of the United Arab Emirates (UAE). International Journal of Auditing, 19(2), 117-130. Consulta: 18 de abril de 2018. https://doi.org/10.1111/ijau.12040

http://www.gao.gov/assets/680/671664.pdf

International Federation of Accountants (IFAC) (2007). Guia para usar los Estándares Internacionales de Auditoría en las pymes. Bogotá: Ecoe Ediciones.

Kagermann, H., Kinney, W., Kuting, K. y Weber, C.-P. (2007). Internal audit handbook. Berlin: Springer. 
Maldonado, M. (2016). Revolución contable: Para la justicia social y la protección ambiental. Quito: Milton K. Maldonado. Consulta: 5 de mayo de 2018. http:// liderazgoyriesgos.com/Revolucion.html

Montenegro, T. M., Oliveira, L. y Lopes, M. S. (2018). $A$ adequabilidade do controle interno no Comando da Aeronáutica: Uma percepção endógena. Innovar, 28(68), 51-66. Consulta: 5 de mayo de 2018. https:// doi.org/10.15446/innovar.v28n68.70471

Nieto, A. (2014). El cumplimiento normativo. En Manual de cumplimiento normativo y responsabilidad penal de las personas jurídicas (pp. 25-48). Valencia: Tirant Lo Blanch.

PricewaterhouseCoopers (2016). Encuesta sobre fraude $y$ delito económico 2016. Resultados en España. Madrid: PwC. Consulta: 5 de mayo de 2018. https:// www.pwc.es/es/publicaciones/transacciones/assets/ pwc-forensic-encuesta-fraude-empresarial-y-delitoeconomico-2016-spain.pdf

Roy, A. y Singer, A. (2006). Reducing corruption in international business: Behavioural, managerial and political approaches. Journal of Economic \& Social Policy, 2(10), 3-24. Consulta: 5 de mayo de 2018. http://dro.deakin.edu.au/view/DU:30020913

Rozas, A. E. (2009). Auditoría forense. Quipukamayoc, 16(32), 67-93. Consulta: 5 de mayo de 2018. https:// doi.org/10.15381/quipu.v16i32.4825

Ryder, N. (2011). Financial crime in the 21st century. Northampton: Edward Elgar.

Sitorus, T. y Scott, D. (2009). Integrated fraud risk factors and robust methodology: A review and comment.
International Journal of Auditing, 13(3), 281-297. Consulta: 7 de mayo de 2018. https://doi.org/10. 1111/j.1099-1123.2009.00398.x

Soto, B. (2016). La auditoría y las TI. El futuro de la auditoría. Revista Contaduría Pública, 44(523), 44-47. Consulta: 7 de mayo de 2018. http://www. anfeca.unam.mx/docs/publicaciones/revistacp/revistacp_201603.pdf

The Institute of Internal Auditors (IIA) (2008). Gestión del riesgo organizacional de fraude: una guia práctica. Florida: The IIA.

The Institute of Internal Auditors (IIA) (2009). Auditoria interna $y$ fraude. Florida: The IIA.

The Institute of Internal Auditors (IIA) (2017a). Definition of Internal Auditing. The Institute of Internal Auditors. Consulta: 7 de mayo de 2018. https:/global.theiia. org/standards-guidance/mandatory-guidance/Pages/ Definition-of-Internal-Auditing.aspx

The Institute of Internal Auditors (IIA) (2017b). Marco internacional para la práctica profesional de la auditoría interna. Altamonte Springs: The Institute of Internal Auditors.

U.S. Government Accountability Office (2015). A framework for managing fraud risks in federal programs. Washington, DC: GAO. Consulta: 20 de abril de 2018.

Fecha de recepción: 24 de enero de 2018 Fecha de aceptación: 12 de abril de 2018 Correspondencia: cdelatorre@uce.edu.ec 